\title{
Rede de apoio social e saúde de idosos pneumopatas crônicos
}

\author{
Social support network and health of elderly individuals \\ with chronic pneumopathies
}

Rafael Barreto de Mesquita ${ }^{1}$

Maria Tereza Aguiar Pessoa Morano ${ }^{2}$

Fátima Luna Pinheiro Landim ${ }^{3}$

Patrícia Moreira Costa Collares ${ }^{4}$

Juliana Maria de Sousa Pinto ${ }^{5}$

${ }^{1}$ Centro de Pesquisa em Ciências da Saúde (CPCS), Centro de Ciências

Biológicas e da Saúde (CCBS), Universidade Norte do Paraná (UNOPAR). Av. Paris 675, Jardim Piza. 86041-120 Londrina PR. rafaelmesquita14@ymail.com

${ }^{2}$ Hospital de Messejana Dr.

Carlos Alberto Studart

Gomes.

${ }^{3}$ Universidade de Fortaleza (UNIFOR).

${ }^{4}$ Faculdades Nordeste (FANOR).

${ }^{5}$ Universidad de Salamanca (USAL)

\begin{abstract}
This study sought to analyze characteristics of the social support network of the elderly with chronic pneumopathies, establishing links with health maintenance/rehabilitation. The assumptions of Social Network Analysis (SNA) methodology were used, addressing the social support concept. A questionnaire and semi-structured interviews, both applied to 16 elderly people attended by a public hospital in Fortaleza-CE, were used for data collection. Quantitative data were processed using the UCINET 6.123, NetDraw 2.38 and Microsoft Excel software programs. In the qualitative analysis, the body of material was subjected to interpretations based on relevant and current theoretical references. Each informant brought an average of 10.37 individuals into the network. Among the 3 types of social support, there was a predominance of informational support given by health professionals. The importance of reciprocity in providing/receiving social support was also noted, as well as the participation of health professionals and the family functioning as social support. The conclusion reached was that the network of the elderly with pneumopathies is not cohesive, being restricted to the personal network of each individual, and that even so, the informants recognize and are satisfied with the social support it provides.
\end{abstract}

Key words Social support, Elderly, Obstructive pneumopathies
Resumo Esse estudo objetivou analisar características estruturais da rede de apoio social de idosos pneumopatas crônicos, traçando relações com a manutenção/recuperação da saúde. Utilizou-se dos pressupostos da metodologia de Análise de Redes Sociais (ARS), focando o conceito de apoio social e suas dimensões. Na coleta dos dados, foram empregados questionário e entrevista semiestruturada, aplicados a 16 idosos assistidos por um hospital público de Fortaleza-CE. Os dados quantitativos foram processados com auxílio dos softwares UCINET 6.123, NetDraw 2.38 e Microsoft Excel. Na analise qualitativa, o corpus constituiu material submetido a interpretações apoiadas em referencial teórico pertinente e atual. Cada informante trazia para a rede uma média de 10,37 indivíduos. Dentre os três tipos de apoio social, houve predominância daquele de informação com procedência nos profissionais de saúde. Ainda se observou a importância da reciprocidade no fornecimento/recebimento de apoio social, o qual funciona com a participação dos profissionais de saúde e da família. Conclui-se que a rede do idoso pneumopata crônico é pouco coesa, restringindo-se à rede pessoal de cada um, e que, mesmo assim, os informantes reconhecem e estão satisfeitos com o apoio social proporcionado por ela.

Palavras-chave Apoio social, Idoso, Pneumopatias obstrutivas 


\section{Introdução}

Pesquisadores em todo o mundo ressaltam amiúde a importância de se estudar a função que o apoio (ou suporte) social desempenha em domínios específicos da vida, considerando realidades contextuais também específicas ${ }^{1-5}$.

A discussão em torno da utilidade do apoio social na vida das pessoas não é recente. Com início no final da década de 1970, diversos são os estudos que buscam explicar a diferença na distribuição de certas enfermidades entre as pessoas e a relação desta com o apoio social recebido ${ }^{6}$. Outros verificam consistente associação entre apoio social e mortalidade ${ }^{7-9}$ ou medidas de saúde, como capacidade funcional, incontinência urinária e problemas visuais entre idosos ${ }^{10}$.

Muito embora não haja consenso quanto ao conceito, advoga-se a ideia de que o apoio social guarda sim uma relação com o bem-estar das pessoas, pois proporciona tanto proteção contra $\mathrm{o}$ aparecimento de doenças, como também a melhoria das dimensões física, mental e emocional ${ }^{1,11}$.

$\mathrm{O}$ apoio social pode ser definido como qualquer informação e/ou auxílio material oferecido por pessoas e/ou grupo(s) que se conhecem e que conferem, como resultado dessa interação, efeitos emocionais e/ou comportamentos positivos $^{12}$. Pode ser classificado, de acordo com a sua função, em emocional, de informação ou material (ou instrumental) ${ }^{13}$.

$\mathrm{O}$ apoio emocional refere-se ao indivíduo ter a sua disposição alguém para falar e desabafar, incluindo atitudes que fomentem o bem-estar afetivo, relacionando-se ao sentimento de estima, de sentir-se querido e respeitado, de poder confiar em alguém. Já o apoio de informação diz respeito ao fornecimento de informações, dar conselhos e orientações. E o apoio material (ou instrumental) vincula-se ao fornecimento de bens e serviços que ajudam a resolver problemas ${ }^{13}$.

$\mathrm{Na}$ realidade das pessoas idosas, os estudos focando o apoio social adquirem destaque pelo fato de os problemas advindos (característicos) com a idade definirem condições mórbidas que tanto podem ter relação com limitações físicas e cognitivas, quanto com o fato de, na sociedade, essa população ser, frequentemente, vítima de segregação e de exclusão social. Na direção desse grupo populacional, as redes de apoio social podem, na maioria das vezes, favorecer uma percepção de estima, de ser amado e valorizado, um sentimento de obrigações mútuas e de pertença a uma coletividade.

Nos últimos anos, tem curso uma grande preocupação no referente às patologias da população idosa, especialmente as crônicas não trans- missíveis e/ou crônico-degenerativas, que mais acometem essa população. Dentre elas estão a hipertensão, o diabetes e as doenças respiratórias. Estas últimas têm significado especial em decorrência da principal sintomatologia que apresentam, a dispneia ("falta de ar"), que repercute enorme e negativamente na vida cotidiana desses indivíduos, impossibilitando-os de realizar um grande número de atividades que rotineiramente praticavam antes do seu surgimento ${ }^{14}$.

Faz-se necessário esclarecer que a Organização Mundial de Saúde (OMS) entende por doença respiratória crônica as enfermidades crônicas de vias aéreas e de outras estruturas dos pulmões ${ }^{15}$. Dentre as mais comuns estão a asma, a doença pulmonar obstrutiva crônica (DPOC), as doenças respiratórias alérgicas, as moléstias pulmonares ocupacionais e a hipertensão pulmonar. Ainda segundo a $\mathrm{OMS}^{15}$, centenas de milhões de pessoas sofrem diariamente de pneumopatias crônicas, sendo que 300 milhões de pessoas têm asma, 80 milhões portam de moderado a grave DPOC e milhões de outras são acometidas de vários outros tipo de doenças respiratórias crônicas, muitas ainda sem diagnóstico.

Com efeito, este estudo objetivou analisar características estruturais da rede social pessoal de idosos pneumopatas crônicos. Os dados quantitativos foram complementados com a avaliação qualitativa das dimensões do apoio social, traçando posterior relação com o favorecimento da manutenção/recuperação da saúde dos idosos.

\section{Métodos}

Trata-se de um estudo descritivo, desenvolvido segundo conceitos e técnicas utilizadas na metodologia de Análise de Redes Sociais (ARS). Essa metodologia é trabalhada por antropólogos, psicólogos sociais e sociólogos desde a década de 1930, e permite privilegiar aspectos distintos das abordagens qualitativa e quantitativa na coleta, na organização e na análise dos dados ${ }^{16,17}$.

Considerando-se as diferentes variáveis de análise da metodologia ARS, passíveis de se empregar na realidade deste estudo, a escolhida foi o apoio social. Esta é muito abrangente, englobando grande conjunto de componentes e aspectos $^{18}$. Adota-se, neste estudo, a classificação mais utilizada por estudiosos do tema, na qual o apoio recebido pode assumir as funções "emocional", "de informação" e "material"13.

Participaram da pesquisa os idosos (acima de 60 anos) pneumopatas crônicos (de acordo com a definição da OMS) que, durante o perío- 
do da coleta de dados, estivessem em tratamento no programa de Reabilitação Pulmonar (RP) do Hospital de Messejana Dr. Carlos Alberto Studart Gomes, localizado na cidade de Fortaleza, Ceará. Esse hospital serviu como espaço físico do estudo e o programa de RP funcionou como meio de acesso aos informantes. O número exato de participantes foi determinado no contato do pesquisador com o campo, de acordo com a disponibilidade de informantes pelo programa de RP e considerando-se o critério de saturação do dado qualitativo ${ }^{19}$.

A coleta dos indicadores foi procedida com o emprego de uma versão adaptada do questionário gerador de nomes e qualificador das relações ${ }^{17}$, nos meses de fevereiro a abril de 2008. Este instrumento permitiu o levantamento das características estruturais das redes e das pessoais dos informantes, assim como a função (emocional, de informação ou material) e a procedência (na família, nos amigos ou nos profissionais de saúde) do apoio social percebido.

Durante o preenchimento do questionário, não foi permitida a marcação de mais de uma opção, e foi pedido aos informantes que manifestassem a escolha de acordo com o vínculo primeiramente estabelecido. Após o preenchimento do questionário, aos informantes foi aplicada a seguinte proposição geradora de discursos: justifique suas opções, comentando a função das pessoas citadas como apoio social. Com o intuito de se garantir a fidedignidade dos dados, os discursos foram gravados e posteriormente transcritos na íntegra.

A análise quantitativa dos dados visou identificar o tamanho da rede, em termos de participações percebidas pelos seus integrantes; a média e o desvio-padrão do número de indicações, da procedência e da função do apoio social informado; e a geração do mapa da rede. Esses levantamentos foram realizados com o auxílio dos seguintes programas computacionais: UCINET $6.123^{20}$, para entrada e manipulação dos dados; NetDraw 2.38, que acompanha o primeiro, para visualização do mapa da rede; e Microsoft Excel, para a identificação dos valores de média e desvio-padrão.

O material qualitativo (as entrevistas transcritas) foi submetido a várias leituras, no intuito de se identificar temas recorrentes, convergências e divergências de falas, ou seja, sistemas classificatórios que permitissem organizar o corpus em tamanhos manuseáveis. Em seguida, esse corpus constituiu material de análises e foi seguido de interpretações apoiadas em referencial teórico pertinente e atual.
Este experimento segue os preceitos éticos estabelecidos na Resolução 196/96, do Conselho Nacional de Saúde/Ministério da Saúde ${ }^{21}$ e foi aprovado pelo Comitê de Ética e Pesquisa do Hospital de Messejana.

\section{Resultados e discussão}

Com base no que permite a ARS, foram aqui apresentados os dados quantitativos da rede, juntamente com a caracterização dos 16 informantes do estudo, e em seguida os dados qualitativos, oriundos dos depoimentos.

\section{Caracterização dos informantes e dados quantitativos da rede de apoio social}

Dos 16 informantes, oito (50\%) eram do sexo masculino, tinham idade média de 69,56 ( \pm 5,05) anos, predominância do estado civil casado(a) (8/50\%), religião católica $(13 / 81,25 \%)$, ocupação aposentado(a) (13/81,25\%), residindo com até duas pessoas (10/62,5\%), tendo mais de 11 anos de doença diagnosticada $(8 / 50 \%)$ e tendo participado do programa de Reabilitação Pulmonar (RP) do Hospital de Messejana (locus de acesso aos informantes) por até três meses $(7 / 43,75 \%)$. Todos se declararam como tendo DPOC, sendo que dois, além desta, informaram possuir, também: um deles, asma crônica, e o outro, cisto pulmonar. A média e o desvio-padrão do tempo durante o qual os indivíduos possuem o diagnóstico da doença foi de 13,37 $( \pm 11,53)$ anos; do tempo em que os indivíduos participaram da RP do referido hospital foi de $16( \pm 24,94)$ meses, e do número de pessoas com quem moram foi de 2,37 ( $\pm 2,09)$ pessoas.

A Figura 1 representa o mapa da rede, organizado com base na sobreposição das redes de apoio social de cada informante. Nela podem ser observados 128 símbolos, representando os membros da rede, designados aqui pela abreviação "AT", referindo-se a "atores sociais", linguagem utilizada na metodologia de ARS. Esse número representa o tamanho da rede social estudada, ou seja, trata-se da agregação de todos os nomes gerados, eliminando-se as repetições e incluídos os nomes de todos os informantes. Os 16 informantes acessados ensejaram o mínimo de três e o máximo de 19 (em média 10,37 $\pm 3,98$ ) nomes compondo a sua rede pessoal de apoio social em saúde.

Se considerarmos que outros estudos ${ }^{22-24}$ revelam redes pessoais com média de participantes 


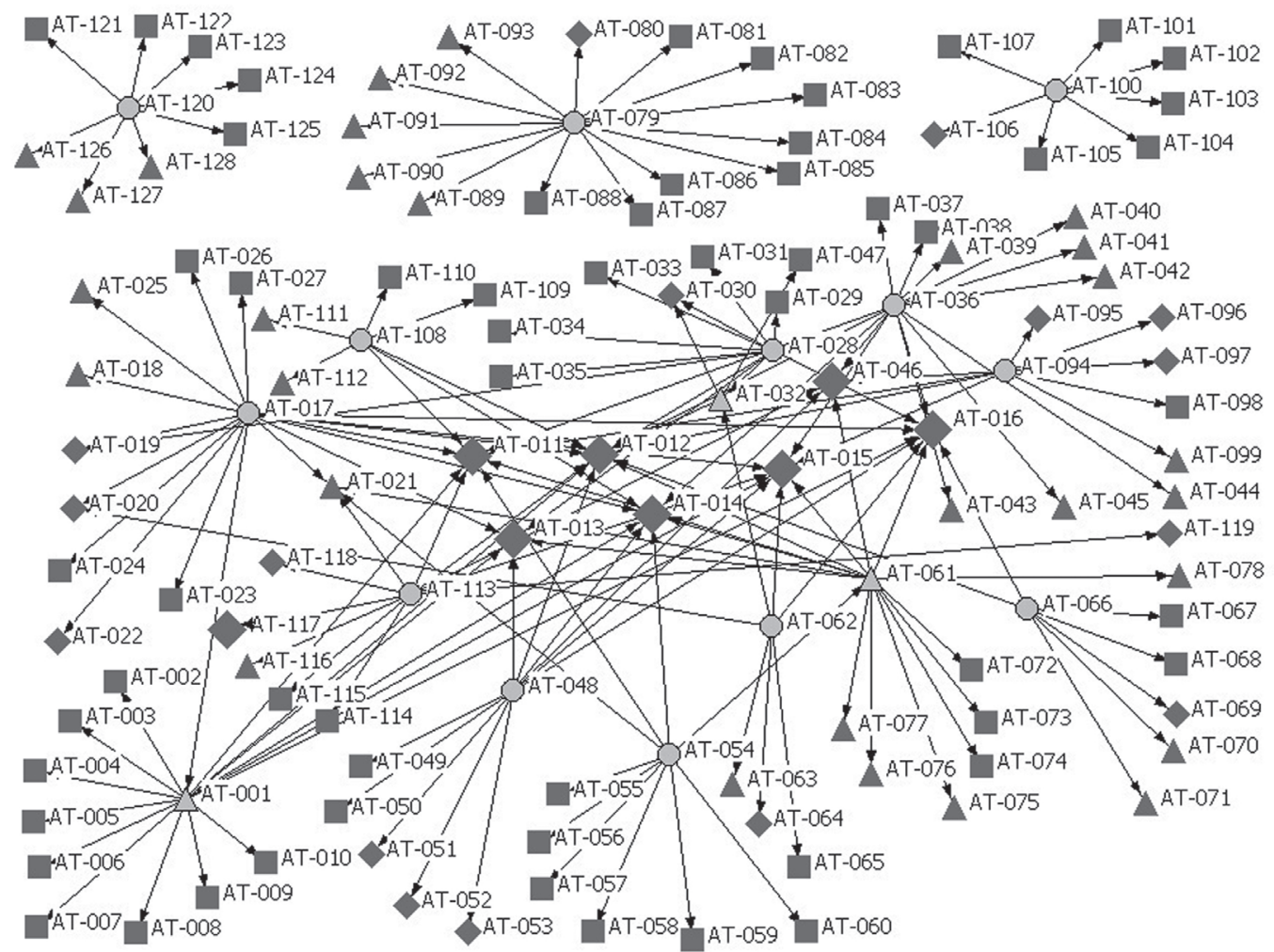

LEGENDAS

Atores citados:

- Procedência na família

A - Procedência nos amigos

- Procedência nos profissionais de saúde

- Profissionais de saúde da Reabilitação Pulmonar
Atores informantes:

- Informantes não citados

$\triangle$ - Informantes citados como amigos

Figura 1. Mapa gerado a partir da rede pessoal de apoio social em saúde dos idosos pneumopatas crônicos. Fortaleza (CE), 2008.

entre quatro e sete membros, pode-se inferir relevância à composição da rede de apoio social dos entrevistados, já que, quanto maior for a rede pessoal de apoio em saúde, maior poderá ser a disponibilidade de ajuda.

Ao analisar a Figura 1, todavia, constata-se que são poucas as conexões estabelecidas entre os atores da rede, traduzindo-se numa rede pouco coesa. Mesquita et al. ${ }^{25}$, analisando uma rede formada por 18 profissionais educadores organizados no sentido da inclusão escolar de crianças com necessidades especiais, encontraram uma lógica parecida de organização social. No seu estudo, eles constataram que esse formato de rede (muitos participantes e pouca coesão) não favorece a manutenção da rede, nem confere a ela poder de resolubilidade, quando consideradas as demandas diárias.

Nesse mesmo estudo, os autores acentuam que essa baixa coesão pode significar pouca exposição, por parte dos indivíduos, aos intercâmbios, às trocas de apoio emocional, de informação e material. Nesta investigação, esse pouco entrosamento pode ter relações tanto com o fato de os indivíduos não verem nos seus colegas uma fonte potencial de apoio social, como também ao fato de que nem todos passaram pela RP no mesmo período. Na verdade, alguns nem sequer se conheceram.

Percebe-se que centralmente, na rede, se localizam os seguintes profissionais de saúde, destacados com símbolos num tamanho maior que 
os demais: AT-011, AT-012, AT-013, AT-014, AT015, AT-016 e AT-046. Esses indivíduos são profissionais do serviço de RP onde os informantes foram acessados, e sua localização no centro da rede decorre do grande número de citações que receberam. AT-011, AT-012, AT-013 e AT-016 são fisioterapeutas ou estagiário de fisioterapia, AT014 é secretária e AT-015 e AT-046 são as médicas do serviço.

No referido programa de RP, a equipe é composta por médicos pneumologistas, fisioterapeutas, terapeuta ocupacional, psicólogo, nutricionista, assistente social, secretária e uma voluntária que ajuda em algumas tarefas técnicas. Importante é destacar que esse é um dos poucos programas, no Brasil, que conta com uma equipe de RP quase completa. Nessa equipe, o fisioterapeuta destaca-se por ser o profissional que está em contato mais próximo com os pacientes, já que é quem acompanha os treinamentos físicos realizados nos dias do programa. O que está associado ao fato de que, no referido programa, é maior o número de profissionais de fisioterapia (dentre profissionais do serviço, bolsistas e estagiários) em relação às outras classes profissionais; talvez se justifique a predominância na citação de fisioterapeutas. Questiona-se, porém, por que os demais profissionais de saúde da RP não foram sequer citados, com exceção da terapeuta ocupacional (AT-117), que recebeu uma citação.

Acredita-se que fazer parte da rede de apoio social em saúde dos pacientes deve ser uma meta vislumbrada pelos profissionais de um serviço de RP, pois, quando forem assim considerados, o grau de confiança dos pacientes aumentará consideravelmente e as suas condutas passarão a ter resultados mais rápidos e eficazes. Nesse propósito, tanto o entrosamento entre a equipe da RP, como aquela entre a equipe e os pacientes e seus familiares devem ser estimulados.

Ainda com base na Figura 1, pode-se observar que os informantes AT-079, AT-100 e AT-120 não se ligaram a nenhum dos profissionais ou pacientes (demais informantes) da RP. Eles referiram como membros da sua rede de apoio social em saúde um universo particular que envolve principalmente familiares e amigos, tendo pouca ou nenhuma participação dos profissionais de saúde. AT-079 há três meses havia recebido alta da RP, enquanto que AT-100 já recebera alta há 15 meses e AT-120 estava participando há apenas um mês desse programa. Os demais informantes do estudo, no momento da coleta dos dados, ou estavam em treinamento na RP ou recebiam visitas domiciliares dos profissionais desse serviço, justificando, talvez, os dados encontrados. Mesquita et al. ${ }^{25}$ apoiaram-se em Moreno ${ }^{26}$ para explicar a situação de atores em situação semelhante à desses. Segundo os autores, eles devem estar vivenciando, provavelmente, a fase de "isolamento orgânico", quando ainda não conhecem os demais participantes do grupo, e muito menos são reconhecidos por eles. Esse fato limita bastante o potencial de trocas no interior da rede, utilizando-se, para suprir essa carência, de apoios externos ao grupo, consolidados antes da sua participação no agrupamento.

A Tabela 1 apresenta a relação entre a procedência e a função do apoio social recebido no interior da rede. Pode-se observar que foram citadas no total 60 (em média $3,75 \pm 2,51$ ) pessoas com procedência na família, 38 (em média $2,37 \pm$ 2,09 ) nos amigos e 68 (em média 4,25 $\pm 3,04$ ) nos profissionais de saúde. Dessas, 46 (em média 2,87 $\pm 1,70$ ) pessoas foram classificadas como apoio emocional, 74 (em média 4,62 $\pm 2,94$ ) como apoio de informação e 46 (em média 2,87 \pm 1,92) como apoio material. No total, foram feitas 166 citações, porém, nesse número estão incluídos nomes repetidos, já que um determinado indivíduo pode funcionar como diferentes apoios para diferentes informantes; e não estão alguns dos informantes acessados, uma vez que nem todos foram citados pelos demais.

Observa-se que a respeito da procedência do apoio social, predominaram os profissionais de

Tabela 1. Relação entre a procedência e a função do apoio social das citações feitas pelos informantes. Fortaleza (CE), 2008.

\begin{tabular}{lcccc}
\hline Função & Procedência & Amigos & Profissionais de saúde & Total \\
\hline Apoio Emocional & 24 & 14 & 8 & 46 \\
Apoio de Informação & 14 & 11 & 49 & 74 \\
Apoio Material & 22 & 13 & 11 & 46 \\
Total & 60 & 38 & 68 & 166 \\
\hline
\end{tabular}


saúde, seguidos da família e dos amigos. Já no que se refere à função do apoio social, houve predominância do apoio de informação relativamente aos demais.

Os dados revelam uma realidade cultural de certo distanciamento da família como apoio social significativo. Sobre o assunto, Robles et al. ${ }^{22}$ investigaram a rede e o apoio social de idosos de baixa renda hospitalizados (em enfermaria) numa cidade do México, a maioria com doenças crônicas (principalmente o Diabetes Mellitus), e verificaram que houve predominância do apoio oriundo da família e do tipo emocional, divergindo deste estudo. No seu estudo, entretanto, os profissionais de saúde parecem não ter sido considerados como fonte de apoio social.

Acredita-se que as diferenças encontradas entre o estudo de Robles e este decorrem, além das diferenças culturais, de pelo menos dois fatores principais: diferentes patologias analisadas e locus e dinâmica de atendimento do serviço (enfermaria ou ambulatório).

Quanto à evidenciada relação feita entre os profissionais de saúde e o apoio de informação, destaca-se que a função dos profissionais de saúde junto aos pacientes idosos com DPOC varia, principalmente, de acordo com a sua especialidade. Uma equipe de RP ideal deve ser composta por pneumologista, fisioterapeuta respiratório, nutricionista, psicólogo, terapeuta ocupacional, enfermeira e assistente social ${ }^{27}$. Não foram realizadas, contudo, análises no sentido de identificar o teor, nem como ocorre o fluxo dessas informações no cuidado prestado.

Mesmo predominando no fornecimento de apoio de informação, também se verificou que os profissionais de saúde fornecem apoio material e, em menor escala, suporte emocional. Acredita-se que é função dos profissionais de saúde fornecer os três tipos de apoio, devendo prevalecer o material, que se traduz nos serviços prestados, e o de informação, mediante informações, conselhos e orientações fornecidas.

\section{Sentimentos e significados atribuídos ao apoio social em saúde}

Nessa etapa, serão apresentados os recortes de discursos que melhor sintetizam a ideia apresentada pela maioria dos informantes, no que diz respeito à função das citações feitas.

Com referência ao apoio emocional, apresenta-se na sequência um trecho do discurso de uma informante, referindo-se ao apoio proporcionado por sua amiga:
É porque nos se achava aqui que não tinha ninguém, nem eu e nem ela, ai ela se encostou a mim e eu me encostei a ela, ai todas necessidades nós tamos no meio sabe, se eu to doente ela ta do meu lado, se ela ta doente, eu ao lado dela [...]. (AT-120).

Observa-se nesta reprodução de fala a importância da reciprocidade no recebimento e fornecimento de apoio social. De acordo com a Teoria da Equidade, dar e receber são os elementos chave do suporte social, e essa troca balanceada, ou seja, esse sentimento de equidade, beneficia psicologicamente ambas as partes e estimula seu sentimento de autovalorização ${ }^{28}$.

Dentre as principais sensações e sinais que preocupam o paciente com DPOC está o "cansaço" (dispneia/fadiga). Quando esse "cansaço" evolui para "uma crise", o pânico, o desespero e o "sufoco que dá medo" tomam conta do corpo e da mente do paciente ${ }^{14}$. Esse sintoma traz grandes repercussões à saúde mental dos pacientes, principalmente pela grande incapacidade que produz no desempenho das atividades cotidianas, tornando-os depressivos e isolados ${ }^{14}$. O entrosamento com outras pessoas, por meio de vínculos sociais, pode, então, contribuir para dar sentido à vida, favorecendo a reorganização da identidade com base nos olhos e nas ações dos outros ${ }^{29}$.

Ainda referente ao apoio emocional, confirmando os dados quantitativos, evidenciaram-se discursos que confirmam a existência e a importância do fornecimento desse tipo de apoio pelos profissionais de saúde:

[...] quando a menina diz que eu to triste, ai ela [uma profissional de saúde] manda dizer que quer falar comigo num sabe, ai quando me vê, vai conversar, me dar um apoio... e quando eu vejo ela, me sinto toda feliz, aquela tristeza vai pra trás. (AT-113).

[...] ela foi quem, fez tudo pra mim parar de fumar sabe, deixar o cigarro. E... me apoiou demais, e eu vendo aquilo, fiquei emocionado com aquilo, e eu senti que realmente ela era uma pessoa que fez tudo por mim né, se num fosse ela eu num tinha deixado de fumar. (AT-032).

Quando foi pedido para que justificassem a função das citações como apoio de informação, a maioria dos informantes produziu discursos semelhantes a esses:

A doutora, se eu chego pra conversar com ela, pedir informação, pedir esclarecimento, ela me dá, ela me auxilia, ela me ajuda pra eu curar a minha doença que eu tenho, me explica como é que é... assim tá ótimo! (AT-062).

[...] ela [uma profissional de saúde] me apoiou desde o momento que nós se conhecemos na reabilitação, ela foi uma criatura que realmente me en- 
sinou até como eu deveria conduzir a minha doença [...]. (AT-061).

A explicação sobre a doença e acerca dos aspectos a ela ligados é fator imprescindível no tratamento dessa enfermidade e na realidade dos programas de RP. O plano educacional deve ser parte integral dos programas de RP e deve incluir informações acerca do autocuidado, da prevenção e do tratamento das exacerbações, sendo essa recomendação classificada como nível 1B por guidelines internacionais ${ }^{30}$.

Embora alguns especialistas na área concordem com a ideia de que devem participar do plano educacional, além dos próprios pacientes, os seus cuidadores e/ou familiares, um informante evidencia a carência de informações sobre a doença para a família:

[...] eles [a sua família] também não tem tanto conhecimento, o conhecimento que eles tem da doença é através de mim mesmo, que eu tenho aprendido lá na reabilitação, eu tenho passado aqui pra eles [...]. (AT-061).

Os profissionais de saúde parecem estar, em algumas situações, ausentes, no que diz respeito ao fornecimento de informações e/ou esclarecimentos dos familiares e/ou cuidadores de doentes crônicos ${ }^{31}$. Uma informante, contudo, evidencia a atenção de um profissional de saúde no esclarecimento acerca da doença à sua filha:

[...] ele [o médico] foi explicar o tratamento à minha filha, ela teve aqui sabe, ele deu muita atenção a ela [...]. (AT-066).

No que diz respeito ao apoio material, surgiram os seguintes discursos:

Olha, ela [a filha] me leva pro hospital, ela me dá o remédio $[\ldots]$ eu digo às vezes que ela não é filha, é mãe [risos] [...]. (AT-113).

Quando eu volto [do hospital, depois de crise de exacerbação da doença], ai eu num tenho condições de tomar banho no banheiro, porque a doença provoca muito cansaço, aí ela [a esposa] me ajuda a eu tomar banho até eu melhorar as condições e voltar a fazer as coisas que eu tenho necessidade. (AT-054).

Dessa forma, percebe-se a importante participação da família, especialmente a nuclear, no fornecimento de apoio material. Dentre as várias consequências da DPOC, está a dependência de cuidados básicos (vestir-se, tomar banho, ir à missa etc.). Essa dependência, contudo, se não vista com "bons olhos" pelos pacientes, pode ser fator depressor e estressor, agravando ainda mais a situação na qual eles se encontram ${ }^{14}$.

À esposa (na realidade dos informantes casados), na maioria das vezes, é incumbido o pa- pel de cuidadora familiar, assumindo, muitas vezes, responsabilidades que nem ela mesma suporta. Essa dependência de cuidados pode prejudicar o seu desempenho em diferentes esferas da sua vida ${ }^{31}$, dentre elas o trabalho, podendo comprometer ainda mais a estabilidade financeira da família ${ }^{14}$.

Todos os informantes foram indagados se estavam satisfeitos com os apoios citados, independentemente da função, e a maioria afirmou que estava bastante satisfeita, produzindo discursos semelhantes aos ora transcritos:

[...] to satisfeita com o apoio dela [da filha], acho que o que ela faz já é demais [risos]. (AT-113).

Com certeza estou satisfeitíssimo, muito, muito mesmo. (AT-061).

A satisfação com a saúde, com o apoio social e com a vida favorece um bem-estar subjetivo que pode funcionar como estratégia eficaz no enfrentamento e na superação das adversidades e dos momentos estressantes do dia a dia. A literatura demonstra que é comum adultos e idosos estarem satisfeitos com as suas relações sociais ${ }^{24}$, achados esses semelhantes aos encontrados neste estudo.

\section{Considerações finais}

Os principais resultados apontam para uma rede de apoio social, em conformidade com os achados da literatura, aparentemente nem tão favorável à saúde dos idosos pneumopatas crônicos, mas que, mesmo assim, parece atender e satisfazer às suas necessidades.

Dizer que, pelo fato de a rede de apoio social em saúde parecer atender às demandas dos pacientes, ela não precisa ser aprimorada, não é totalmente verdade. $\mathrm{O}$ aumento, em número e qualidade, dos membros da rede de apoio social em saúde dos pacientes deve ser sempre estimulado, principalmente pelos profissionais de saúde, que também devem objetivar fazer parte dessa rede. Esse propósito parece já ser posto em prática pelos profissionais de saúde citados neste estudo, uma vez que predominaram na procedência do apoio social, fornecendo, em maior escala, apoio de informação. Isso lhes confere uma grande responsabilidade, pois é com eles que os pacientes irão contar para obter informações, conselhos e orientações para lidar com a sua saúde, sendo esse um "poder" que pode e deve ser administrado com muita cautela.

Visando à manutenção dos benefícios proporcionados pelo apoio social fornecido pela RP, 
acredita-se que, assim como se faz necessário um nível mínimo de atividade física após a alta da RP, um nível mínimo de apoio social também deve ser garantido.

A reciprocidade no fornecimento/recebimento de apoio social e a participação da família, além dos profissionais de saúde, também se fizeram destacar, sendo também fatores favorecedores de uma melhor saúde (física, mental e social) da população investigada.

\section{Colaboradores}

Este artigo é fruto do Trabalho de Conclusão de Curso de RB Mesquita, sob orientação de MTAP Morano e coorientação de FLP Landim. PMC Collares e JMS Pinto contribuíram na análise dos dados, redação e revisão final do artigo.
A vista dos achados, verificou-se que é de grande importância a utilidade da rede de apoio social na saúde dos idosos pneumopatas crônicos, população esta fragilizada pela idade, doença e cronicidade. Estudos como este são pontos fundamentais no estabelecimento de estratégias de cuidados em saúde a populações como a aqui apresentada, uma vez que instiga a se pensar em políticas públicas específicas a fim de favorecer uma assistência especializada.

\section{Referências}

1. Cassell EJ. An epidemiological perspective of psychosocial factors in disease etiology. Am J Public Health 1974; 64(11):1040-1043.

2. Kaplan GA, Salonen JT, Cohen RD, Brand RJ, Syme SL, Puska P. Social connections and mortality from all causes and from cardiovascular disease: prospective evidence from Eastern Finland. Am J Epidemiol 1988; 128(2):370-380.

3. Vogt TM, Mullooly JP, Ernst D, Pope CR, Hollis JF. Social networks as predictors of ischemic heart disease, cancer, stroke and hypertension: incidence, survival and mortality. J Clin Epidemiol 1992; 45(6): 659-666.

4. Fratiglione L, Wang HX, Ericsson K, Maytan M, Winblad B. Influence of social network on occurrence of dementia: a community-based longitudinal study. Lancet 2000; 355(9212):1315-1319.

5. Faerstein E, Chor D, Griep RH, Alves MGM, Werneck GL, Lopes CS. Aferição da pressão arterial: experiência de treinamento de pessoal e controle de qualidade no Estudo Pró-Saúde. Cad Saude Publica 2006; 22(9):1997-2002.

6. Rosa TEC, Benício MHD, Alves MCGP, Lebrão ML. Aspectos estruturais e funcionais do apoio social de idosos do Município de São Paulo, Brasil. Cad Saude Publica 2007; 23(12):2982-2992.

7. Rahman MO. Age and gender variation in the impact of household structure on elderly mortality. Int J Epidemiol 1999; 28(3):485-491.

8. Davis MA, Moritz DJ, Neuhaus JM, Barclay JD, Gee L. Living arrangements, changes in living arrangements, and survival among community dwelling older adults. Am J Public Health 1997; 87(3):371-377.

9. Yasuda N, Zimmerman SI, Hawkes W, Fredman L, Hebel JR, Magaziner J. Relation of social network characteristics to 5-year mortality among youngold versus old-old white women in an urban community. Am J Epidemiol 1997; 145(6):516-523.

10. Litwin H. Social network type and morale in old age. Gerontologist 2001; 41(4):516-524. 
11. Landim FLP, Comaru JL, Mesquita RB, Collares PM. Redes sociais informais no cotidiano das famílias de uma comunidade da periferia de Fortaleza. Cogitare Enferm 2006; 11(1):16-23.

12. Valla VV. Redes sociais, poder e saúde à luz das classes populares numa conjuntura de crise. Interface Comun Saúde Educ 2000; 4(7):37-56.

13. Barrón AI. Apoyo social: aspectos teóricos y aplicaciones. Madrid: España Editores; 1996.

14. Pinto JMS, Vieira LJS, Nations MK. Sopro de vida: experiência com a Doença Pulmonar Obstrutiva Crônica na pobreza urbana de Fortaleza, Ceará, Brasil. Cad Saude Publica 2008; 24(12):2809-2818.

15. World Health Organization (WHO). Chronic respiratory diseases. [site na Internet]. [acessado $2007 \mathrm{fev} 28$ ]. Disponível em: http://www.who.int/respiratory/en/

16. Freeman LC. Some antecedents of social network analysis. Connections 1996; 19(1):39-42.

17. Silva MCM. Redes sociais intraorganizacionais informais e gestão: um estudo nas áreas de manutenção e operação da planta HYCO-8, Camaçari-BA [dissertação]. Salvador (BA): Universidade Federal da Bahia; 2003.

18. Ribeiro JLP. Escala de Satisfação com o Suporte Social (ESSS). Aná Psicológica 1999; 17(3):547-558.

19. Bosi MLM, Mercado FJ. Pesquisa qualitativa de serviços de saúde. Petrópolis: Vozes; 2004.

20. Borgatti SP, Everett MG, Freeman LC. UCINET Version 6.123. Natick: Analytic Technologies; 2006.

21. Brasil. Ministério da Saúde. Resolução CNS no. 196, de 10 de outubro de 1996. Aprova diretrizes e normas técnicas regulamentadoras de pesquisas envolvendo seres humanos. Diário Oficial da União 1996; out 16.

22. Robles L, Curiel GR, García LMC, Coles LC, Medrano MSG, González MS. Redes y apoyo social en ancianos enfermos de escasos recursos en Guadalajara, México. Cad Saude Publica 2000; 16(2):557-560.
23. Wenger GC. Review of findings on support networks of older Europeans. J Cross Cult Gerontol 1997; 12(1):1-21.

24. Resende MC, Bones VM, Souza IS, Guimarães NK. Rede de relações sociais e satisfação com a vida de adultos e idosos. Psicol Am Lat [periódico na Internet]. 2006 [acessado 2008 nov 03];5(5):1-20. Disponível em: http://psicolatina.org/Cinco/index.html

25. Mesquita RB, Landim FLP, Collares PM, Luna CG. Análise de redes sociais informais: aplicação na realidade da escola inclusive. Interface Comunic Saúde Educ 2008; 12(26):549-562.

26. Moreno JL. Quem sobreviverá?: fundamentos da sociometria, psicoterapia de grupo e sociodrama. Goiânia: Dimensões; 1994.

27. Tarantino AB. Doenças Pulmonares. $5^{\mathrm{a}}$ ed. Rio de Janeiro: Guanabara Koogan; 2002.

28. Ramos MP. Apoio social e saúde entre idosos. Sociologias 2002; 4(7):156-175.

29. Andrade GRB, Vaitsman J. Apoio social e redes: conectando solidariedade e saúde. Cien Saude Colet 2002; 7(4):925-934.

30. Ries AL, Emery CF, Mahler DA, Bauldoff GS, Carlin BW, Casaburi R, Emery CF, Mahler DA, Make B, Rochester CL, Zuwallack R, Herrerias C. Pulmonary rehabilitation: joint ACCP/AACVPR evidencebased clinical practice guidelines. Chest 2007; 131(Supl. 5):4-42.

31. Marques AKMC, Landim FLP, Collares PM, Mesquita RB. Apoio social na experiência do familiar cuidador. Cien Saude Colet 2008; 16(Supl. 1):945-955.

Artigo apresentado em 22/05/2010

Aprovado em 06/09/2010

Versão final apresentada em 15/09/2010 